\title{
AVALIAÇÃo DO ÓleO DE SOJA SUBMETIDO AO PROCESSO DE FRITURA DE ALIMENTOS DIVERSOS
}

\author{
Evaluation of soybean oil submitted to the frying process of varied foods
}

\author{
Neuza Jorge ${ }^{1}$, Camila Janieri ${ }^{2}$
}

\begin{abstract}
RESUMO
O presente trabalho teve como objetivo avaliar o estado de degradação do óleo de soja utilizado em frituras descontínuas. A qualidade do óleo foi monitorada durante o processo de fritura mediante análises físico-químicas como dienos conjugados (\%) e ácido tiobarbitúrico - TBA (mmoles $\mathrm{g}^{-1}$ ) e determinação de compostos polares totais (\%). Verificou-se que os valores de dienos aumentaram significativamente com o tempo e atingiram 1,87\% após 15 horas para a Fritadeira I e 1,76\% após 22,5 horas para a Fritadeira II. Os índices de TBA alcançaram ao final do processo, valores de 0,0265 e $0,0210 \mathrm{mmoles} \mathrm{g}^{-1}$ para as Fritadeiras I e II, respectivamente. As porcentagens de compostos polares totais apresentaram aumento de seis e oito vezes em relação à amostra original partindo de valores de 5,9\% e 3,7\% e atingindo valores de 36,8\% e 29,2\%, para as Fritadeiras I e II, após 15 e 22,5 horas de aquecimento, respectivamente. Assim, conclui-se que houve um aumento nas alterações do óleo de soja com o tempo de fritura, em ambas as fritadeiras. O óleo utilizado na Fritadeira I foi o que apresentou maior grau de alteração, quando comparado com o óleo da Fritadeira II, apesar do menor tempo de aquecimento. Considerando-se os limites permitidos pelas legislações de alguns países como compostos polares inferiores a 25\%, os óleos das duas fritadeiras já encontravam sem condições de uso no momento do descarte.
\end{abstract}

Termos para indexação: Óleo de soja, processo de fritura, análises físico-químicas, TBA.

\section{ABSTRACT}

The main objective of this study was to evaluate the degree of degradation of the soybean oil used in frying processes. The quality of the oil during the frying process was evaluated by means of physical-chemical analyses, such: conjugated dienes (\%) and TBA index (mmoles $\mathrm{g}^{-1}$ ), and determination of total polar compounds (\%). It was observed that the values of conjugated dienes increasing significantly with time until $1.87 \%$ after 15 hours for fry pans I and $1.76 \%$ after 22.5 hours for fry pans II. The TBA indexes reached at the end the frying process values of 0.0265 and 0.0210 mmoles $\mathrm{g}^{-1}$ for fry pans I and II, respectively. The percentages of total polar compounds have shown an increase of six and eight times in relation to the original sample starting from $5.9 \%$ and $3.7 \%$ and reaching values of $36.8 \%$ and $29.2 \%$, for fry pan I and II, after 15 and 22.5 hours of heating, respectively. It has been concluded that the alterations of the soybean oil increased as the time of frying increased, for both fry pans. The oil used in fry pan I showed greater alteration than the that observed in the oil used in fry pan II, in spite of the time of heating being shorter. Taking into consideration that the levels of polar compounds should be less than $25 \%$ according to the legislation of several countries, the oils used in both fry pans were already inadequate conditions at the moment of disposal.

Index terms: soybean oil, frying process, physical-chemical analyses, TBA.

\section{(Recebido para publicação em 26 de novembro de 2003 e aprovado em 27 de junho de 2005)}

\section{INDRODUÇÃO}

O processo de fritura desenvolve características de odor, sabor, cor e textura que tornam os alimentos mais atraentes para o consumo. Além disso, considerando que parte do óleo utilizado como meio de transferência de calor é absorvida pelo alimento, tornando-se um ingrediente do produto, verifica-se a necessidade do uso de um meio de fritura de alta qualidade e a manutenção desta por períodos mais longos possíveis (CELLA et al., 2002).

Durante o processo de fritura ocorre alterações termoxidativas que alteram a qualidade do óleo. Sendo assim, a avaliação da alteração e a identificação dos compostos formados durante a fritura de alimentos é de grande interesse para os consumidores, uma vez que esse óleo provavelmente fará parte da sua dieta diária.

Estudos com óleos aquecidos por longos períodos, sob temperaturas elevadas, demonstraram que os produtos resultantes contêm mais de $50 \%$ de compostos polares, que são os produtos de degradação dos triglicerídios (polímeros, dímeros, ácidos graxos oxidados, diglicerídios e ácidos graxos livres). Os óleos com elevados teores de compostos polares provocaram severas irritações do trato gastrointestinal, diarréia, redução no crescimento e, em alguns casos, morte de animais em laboratório (BILLEK, 1985).

Há uma variedade de métodos analíticos que são utilizados para a avaliação dos óleos e gorduras de fritura (STEVENSON et al., 1984; WHITE, 1991). Por um lado, dispõe-se de métodos que quantificam de forma direta os

'Professora Drà. do Departamento de Engenharia e Tecnologia de Alimentos, Instituto de Biociências, Letras e Ciências Exatas/Universidade Estadual Paulista - Rua Cristóvão Colombo, 2265 - Jardim Nazareth - 15054-000 - São José do Rio Preto, SP - njorge@ibilce.unesp.br ${ }^{2}$ Aluna de Iniciação Científica - Bolsista CNPq/PIBIC. 
compostos de alteração originados, entre os quais destacam a determinação de compostos polares e a determinação específica dos compostos de polimerização, oxidação e hidrólise (DOBARGANES et al., 1988), relacionados com os três tipos de alterações mais importantes que ocorrem no processo de fritura. Por outro lado, encontram-se os tradicionais índices físico-químicos que são largamente utilizados para medir as diferentes alterações produzidas no óleo aquecido, tais como os índices de acidez, peróxidos, iodo, refração, métodos colorimétricos, viscosidade, dienos e trienos conjugados e outros (MASSON et al., 1997).

Existem regulamentações em alguns países como Bélgica, França, Alemanha, Suíça, Holanda, Estados Unidos e Chile, sobre as condições em que um óleo utilizado para fritura deve ser descartado, mas no Brasil, como em muitos outros países, não existem leis e regulamentos que estabeleçam limites para as alterações nesses óleos (FIRESTONE et al., 1991). De modo geral, estima-se que óleos deteriorados pelo processo de fritura devem ser descartados quando o seu teor de compostos polares se encontrar acima de 25\% (FIRESTONE et al., 1991). Outro parâmetro considerado é a porcentagem de ácidos graxos livres, para os quais, as legislações estabelecem limites máximos em torno de 1 e 2,5\%. Outros parâmetros como ponto de fumaça, que deve ser superior a $170^{\circ} \mathrm{C}$, formação de espuma, usos de antioxidantes e antiespumantes também podem ser considerados nas legislações e/ou recomendações (MASSON et al., 1999; PAUL \& MITTAL, 1997). Devido à situação, nos últimos anos, muitos esforços têm sido feitos para se desenvolver métodos analíticos de quantificação dos compostos originados durante o processo, já que os índices clássicos parecem ser ineficientes para óleos e gorduras de origem desconhecida. Portanto, é necessário se dispor de métodos de controle das alterações produzidas em óleos durante o processo de fritura, assim como buscar critérios objetivos que definam quando um óleo deve ser descartado.

Tais circunstâncias mostram a importância de se conhecer o comportamento de óleos vegetais utilizados em restaurantes de um modo geral e seu estado de deterioração, uma vez que estes farão parte da dieta diária da população.

O presente trabalho teve como objetivo avaliar, por meio de análises físico-químicas, o estado de degradação do óleo de soja durante frituras de alimentos variados, em um restaurante universitário, e estabelecer o momento mais adequado para o seu descarte.

\section{MATERIAL E MÉTODOS}

\section{Amostras}

O restaurante universitário do Instituto de Biocências, Letras e Ciências Exatas da Universidade Estadual Paulista, o qual fornece diariamente cerca de 300 refeições, utiliza duas fritadeiras elétricas de aço inox, marca Fritomac, com capacidade para 23 litros, operando com uma relação superfície/volume de $0,5 \mathrm{~cm}^{-1}$. Juntamente com o óleo é adicionada uma solução salina que fica em contato direto com o mesmo, formando uma camada inferior, na qual se depositam resíduos dos alimentos fritos.

O óleo de soja, em cada fritadeira, foi utilizado por 2,5 horas diárias a uma temperatura média de $170 \pm 5^{\circ} \mathrm{C}$. As Fritadeiras I e II foram utilizadas durante períodos de 7 e 9 dias, perfazendo um total de 15 e 22,5 horas de aquecimento, respectivamente. Após estes períodos de fritura, o óleo foi descartado. Ao final de cada dia os óleos eram filtrados e as soluções salinas eram trocadas por novas, havendo reposição de óleo novo para manter o nível das fritadeiras quando necessário.

As amostras com $20 \mathrm{~mL}$ de óleo de fritura foram coletadas em recipientes de vidro âmbar e, em seguida, armazenadas à temperatura de aproximadamente $-20^{\circ} \mathrm{C}$ para evitar posteriores alterações oxidativas. As amostras foram descongeladas apenas no momento das análises, as quais foram realizadas em duplicatas.

\section{Determinações analíticas}

Dienos conjugados. Empregou-se o método AOCS Ti 1a-64 (AOCS, 1993), que determina dienos conjugados de ligações insaturadas, expressos como porcentagem de ácidos dienóicos conjugados. Foi utilizado Espectrofotômetro, marca SHIMADZU, modelo UV Mini 1240.

Índice de ácido tiobarbitúrico - TBA. O teste de TBA é uma medida da rancidez e baseia-se na reação do ácido 2-tiobarbitúrico com os aldeídos formados na oxidação de lipídios. Foi utilizado o método das Normas Analíticas do Instituto Adolfo Lutz (1985). As medidas de TBA foram feitas no espectrofotômetro, marca SHIMADZU, modelo UV Mini 1240.

Compostos polares totais. A determinação de compostos polares mediante cromatografia em coluna foi realizada conforme o método cromatográfico proposto por Waltking \& Wessels (1981) com uma pequena modificação: o uso de hexano:éter etílico 90:10, ao invés de 87:13, para produzir melhor separação da fração não polar (DOBARGANES et al., 1984). 


\section{Análise estatística}

Para determinar a influência do tempo de fritura sobre a alteração do óleo submetido ao processo de fritura, para cada fritadeira, os resultados obtidos das determinações analíticas, em duplicata, foram submetidos à análise de variância no delineamento inteiramente casualizado e as diferenças entre as médias foram testadas a $5 \%$ de probabilidade, pelo teste de Tukey (GOMES, 2000), com utilização do programa ESTAT.

\section{RESULTADOS E DISCUSSÃO}

As Tabelas 1 e 2 apresentam os períodos de tempo em que o óleo foi coletado ao longo do processo de fritura, os alimentos fritos e a quantidade de óleo novo adicionada nas Fritadeiras I e II, respectivamente.

A qualidade do óleo foi monitorada durante o processo de fritura por meio de análises espectrofotométricas como dienos conjugados (\%) e TBA (mmoles g ${ }^{-1}$ de óleo) e por análises cromatográficas através da determinação de compostos polares totais (\%), cujos resultados das determinações para as Fritadeiras I e II se encontram nas Tabelas 3 e 4, respectivamente.

Os valores iniciais de dienos conjugados foram 0,42 e $0,60 \%$ atingindo valores de 1,87 e $1,76 \%$ ao final de 15 e 22,5 horas para as Fritadeiras I e II, respectivamente. Observando-se as Tabelas 3 e 4, verifica-se que houve um aumento significativo na porcentagem de dienos conjugados com o decorrer do tempo para as duas fritadeiras empregadas no processo.

Cella et al. (2002), em estudo do comportamento do óleo de soja em fritura por imersão com diferentes alimentos de origem vegetal (mandioca, couve-flor, abobrinha e batata), constataram que o acréscimo do tempo de aquecimento promoveu um aumento nos valores de dienos conjugados.

A avaliação de dienos conjugados tem uma clara significação nas análises de óleos e gorduras de fritura. Como pode ser observado na Tabela 3, o óleo de soja da Fritadeira I apresentou maior grau de alteração que o da Fritadeira II, isso pode ser atribuído ao tipo de alimento frito, tais como, carnes e produtos empanados. Em estudos realizados, Lake \& Scholes (1997) demonstraram que óleos nos quais foram fritos produtos como carnes e peixes, apresentaram valores de dienos conjugados superiores a produtos de origem vegetal, como por exemplo batata frita.

Os valores encontrados neste trabalho foram similares aos descritos por Del Ré (2003) e Masson et al. (1997) ao estudarem óleos poliinsaturados obtidos de estabelecimentos comerciais, utilizados em frituras de batatas, cujos resultados apresentaram-se dentro do intervalo 0,5 a $2,6 \%$.

Os índices de TBAs iniciais foram 0,0015 e 0,0008 mmoles $\mathrm{g}^{-1} \mathrm{e}$ atingiram valores de 0,0265 e 0,0210 mmoles $\mathrm{g}^{-1}$ ao final de 15 e 22,5 horas para as Fritadeiras I e II, respectivamente (Tabelas 3 e 4). Os valores obtidos para os índices de TBA foram inferiores aos encontrados por Lake \& Scholes (1997) em experimento com frituras em óleos vegetais, devido provavelmente, às condições do processo serem diferentes, como temperatura mais elevada e maior tempo de aquecimento. Em outro experimento similar, Zhang \& Addis (1990), determinando o índice de TBA de uma mistura de $90 \%$ de sebo e $10 \%$ de óleo de algodão utilizada para fritura de batata chips a aproximadamente $170^{\circ} \mathrm{C}$, encontraram valores na faixa de 0,004 a 0,010 mmoles $^{-1}$.

TABELA 1 - Esquema geral de utilização da Fritadeira I durante o processo de fritura de alimentos de origem animal e vegetal empanados e não empanados.

\begin{tabular}{|c|c|c|c|c|}
\hline Dia & $\begin{array}{c}\mathbf{t f} \\
\text { (horas) }\end{array}$ & $\begin{array}{c}\text { TF } \\
\text { (horas) }\end{array}$ & $\begin{array}{c}\text { Reposição de óleo } \\
\text { (litros) }\end{array}$ & Alimentos Fritos \\
\hline $1^{\circ}$ & 2,5 & 2,5 & - & Coxinha, risoles, berinjela empanada. \\
\hline $2^{\circ}$ & 2,5 & 5,0 & - & Coxinha, quibe, peixe à milanesa. \\
\hline $3^{\circ}$ & 2,5 & 7,5 & 2 & $\begin{array}{l}\text { Bolinho de legumes, filé de frango empanado, } \\
\text { mandioca, coxinha, Quibe, batata palha. }\end{array}$ \\
\hline $4^{\circ}$ & 2,5 & 10,0 & 2 & Quiabo, quibe, coxinha, risoles. \\
\hline $5^{\circ}$ & 2,5 & 12,5 & - & Coxinha, quibe, couve-flor à dorê. \\
\hline $6^{0} *$ & - & 12,5 & - & - \\
\hline $7^{\circ}$ & 2,5 & 15,0 & - & Bife à milanesa. \\
\hline
\end{tabular}

tf - tempo de fritura por dia, TF - tempo acumulado de fritura, $*$ - dia sem fritura. 
TABELA 2 - Esquema geral de utilização da Fritadeira II durante o processo de fritura de alimentos de origem animal e vegetal empanados e não empanados.

\begin{tabular}{|c|c|c|c|c|}
\hline Dia & $\begin{array}{c}\mathbf{t f} \\
\text { (horas) }\end{array}$ & $\begin{array}{c}\text { TF } \\
\text { (horas) }\end{array}$ & $\begin{array}{c}\text { Reposição de óleo } \\
\text { (litros) }\end{array}$ & Alimentos Fritos \\
\hline $1^{\circ}$ & 2,5 & 2,5 & - & Abobrinha à dorê e batata doce. \\
\hline $2^{\circ}$ & 2,5 & 5,0 & - & Bolinho de legumes, batata frita e polenta frita. \\
\hline $3^{\circ}$ & 2,5 & 7,5 & - & Bolinho de legumes, quibe e risoles. \\
\hline $4^{\circ}$ & 2,5 & 10,0 & 2 & Berinjela empanada e peixe à dorê. \\
\hline $5^{\circ}$ & 2,5 & 12,5 & - & Mandioca, coxinha, quibe e risoles. \\
\hline $6^{\circ}$ & 2,5 & 15,0 & - & $\begin{array}{l}\text { Couve-flor à dorê, coxinha e salsicha à } \\
\text { milanesa. }\end{array}$ \\
\hline $7^{\circ}$ & 2,5 & 17,5 & 2 & $\begin{array}{l}\text { Bolinho de legumes, batata doce, quiabo, } \\
\text { coxinha e risoles. }\end{array}$ \\
\hline $8^{\circ}$ & 2,5 & 20,0 & - & Berinjela à dorê, batata frita, risoles e coxinha. \\
\hline $9^{\circ}$ & 2,5 & 22,5 & - & $\begin{array}{l}\text { Abobrinha e frango à dorê, bolinho de queijo, } \\
\text { coxinha e quibe. }\end{array}$ \\
\hline
\end{tabular}

tf - tempo de fritura por dia, $\mathrm{TF}$ - tempo acumulado de fritura.

TABELA 3 - Tempo de uso (dia e horas acumulados) da Fritadeira I e respectivos valores médios de dienos conjugados (DC), ácido tiobarbitúrico (TBA) e compostos polares totais (CPT).

\begin{tabular}{|c|c|c|c|c|}
\hline \multicolumn{2}{|c|}{ Tempo } & \multirow{2}{*}{$\begin{array}{r}\text { DC } \\
(\%)\end{array}$} & \multirow{2}{*}{$\begin{array}{c}\text { TBA } \\
\left(\mu \text { moles g }^{-1}\right)\end{array}$} & \multirow{2}{*}{$\begin{array}{l}\text { CPT } \\
(\%)\end{array}$} \\
\hline (dia) & (horas) & & & \\
\hline 0 & 0 & $0,42^{\mathrm{d}}$ & $0,0015^{\mathrm{c}}$ & $5,9^{\mathrm{e}}$ \\
\hline 1 & 2,5 & $0,58^{\mathrm{d}}$ & $0,0100^{\mathrm{b}}$ & $18,9^{\mathrm{d}}$ \\
\hline 2 & 5 & $0,90^{\mathrm{c}}$ & $0,0140^{\mathrm{b}}$ & \\
\hline 3 & 7,5 & $1,06^{\mathrm{c}}$ & $0,0140^{\mathrm{b}}$ & $21,9^{c}$ \\
\hline 4 & 10 & $1,60^{\mathrm{ab}}$ & $0,0140^{\mathrm{b}}$ & \\
\hline 5 & 12,5 & $1,55^{\mathrm{b}}$ & $0,0140^{\mathrm{b}}$ & $24,4^{\mathrm{b}}$ \\
\hline 6 & 12,5 & $1,71^{\mathrm{ab}}$ & $0,0150^{\mathrm{b}}$ & \\
\hline 7 & 15 & $1,87^{\mathrm{a}}$ & $0,0265^{\mathrm{a}}$ & $36,8^{\mathrm{a}}$ \\
\hline
\end{tabular}

a, b... (coluna) - médias seguidas de mesma letra não diferem entre si pelo teste de Tukey ( $p>0,05)$.

Vários fatores podem afetar a análise da reação do ácido tiobarbitúrico, tais como o tipo de óleo vegetal, forma de envase e armazenamento das amostras, além da técnica aplicada e a presença de substâncias que diminuem a oxidação.

De acordo com as Tabelas 3 e 4, verifica-se que os índices de TBA aumentaram significativamente com o tempo para ambas as Fritadeiras. Porém, a última amostra da Fritadeira I apresentou maior valor de índice de TBA que a última da Fritadeira II, apesar do menor tempo de aquecimento, o que pode ser atribuído ao tipo de alimento frito, como bife à milanesa.

Os resultados de compostos polares totais obtidos da Fritadeira I, para as amostras inicial e alteradas após o primeiro, terceiro, quinto e sétimo dias de fritura correspondentes a 2,5, 7,5, 12,5 e 15 horas e, da Fritadeira II, para as amostras inicial e alteradas após o primeiro, terceiro, quinto, sétimo e nono dias de fritura correspondentes a $2,5,7,5,12,5,17,5$ e 22,5 horas, encontram-se nas Tabelas 3 e 4. 
Observa-se que as porcentagens de compostos polares das amostras do óleo de soja iniciais foram de 5,9 e $3,7 \%$ e atingiram valores de 36,8 e 29,2 , para amostras alteradas após 15 e 22,5 horas de aquecimento, correspondentes às Fritadeiras I e II, respectivamente, representando aumento próximo de seis e oito vezes em relação às amostras iniciais. Verifica-se para cada fritadeira, que os valores de compostos polares diferiram significativamente entre si pelo teste de Tukey. Considerando-se os limites permitidos pela legislação de alguns países como compostos polares inferiores a $25 \%$, os óleos das duas fritadeiras já encontravam-se sem condições de uso no momento do descarte, ou seja, após 15 e 22,5 horas de aquecimento. Ainda, observa-se pela Tabela 3 que no tempo de fritura de 12,5 horas, a porcentagem de compostos polares totais na Fritadeira I atingiu valor próximo de $25 \%$, ou seja, $24,4 \%$ e, na Fritadeira II, a porcentagem de compostos polares totais ultrapassou o limite de $25 \%$ no tempo de fritura correspondente a 17,5 horas, com valor de $26,7 \%$.

O valor de compostos polares totais para a última amostra da Fritadeira I $(36,8 \%)$ foi superior ao valor encontrado para a última amostra da Fritadeira II $(29,2 \%)$, apesar do tempo de aquecimento da primeira ter sido 7,5 horas inferior. Isso pode ser explicado pelo maior emprego de alimentos gordurosos e empanados na Fritadeira I, tais como bife à milanesa, peixe, produtos cárneos e empanados em geral, enquanto que na Fritadeira II houve a predominância de produtos de origem vegetal.

De acordo com dados obtidos e apresentados anteriormente, verifica-se que as amostras de óleo com maior porcentagem de compostos polares foram as que também apresentaram maiores índices de dienos conjugados e TBA.

Em estudos realizados por Pozo-Díez (1995), concluiu-se que a velocidade de degradação é proporcional ao tempo de fritura, devido ao aumento dos compostos polares durante o processo de fritura, o que coincide com outros autores (CUESTA et al., 1991; MASSON et al., 1997; DEL RÉ, 2003). Comportamento similar foi observado nesse estudo, podendo ser comprovado pelas altas correlações obtidas entre tempo de fritura e compostos polares totais para ambas as fritadeiras empregadas nos processos.

Na Tabela 5 apresentam-se os coeficientes de correlação linear entre as determinações físico-químicas realizadas no óleo de soja e o tempo de fritura, para as Fritadeiras I e II. Observa-se para ambas fritadeiras, que as maiores correlações ocorreram entre dienos conjugados $\mathrm{x}$ tempo de fritura $(0,98)$, seguidas das correlações entre compostos polares e TBA x tempo de fritura, cujos valores foram 0,90 e 0,80 , respectivamente.

TABELA 4 - Tempo de uso (dia e horas acumulados) da Fritadeira II e respectivos valores médios de dienos conjugados (DC), ácido tiobarbitúrico (TBA) e compostos polares totais (CPT).

\begin{tabular}{|c|c|c|c|c|}
\hline \multicolumn{2}{|c|}{ Tempo } & \multirow{2}{*}{$\begin{array}{l}\text { DC } \\
(\%)\end{array}$} & \multirow{2}{*}{$\begin{array}{c}\text { TBA } \\
\left(\mu \text { moles } \mathbf{g}^{-1}\right)\end{array}$} & \multirow{2}{*}{$\begin{array}{r}\text { CPT } \\
(\%)\end{array}$} \\
\hline (dia) & (horas) & & & \\
\hline 0 & 0 & $0,60^{g}$ & $0,0008^{\mathrm{e}}$ & $3,7^{\mathrm{c}}$ \\
\hline 1 & 2,5 & $0,65^{\mathrm{fg}}$ & $0,0055^{\mathrm{e}}$ & $17,6^{\mathrm{b}}$ \\
\hline 2 & 5 & $0,68^{\mathrm{fg}}$ & $0,0120^{\mathrm{d}}$ & \\
\hline 3 & 7,5 & $0,79^{\mathrm{efg}}$ & $0,0130^{\mathrm{cd}}$ & $19,6^{\mathrm{b}}$ \\
\hline 4 & 10 & $0,91^{\text {def }}$ & $0,0150^{\mathrm{cd}}$ & \\
\hline 5 & 12,5 & $1,04^{\text {cde }}$ & $0,0150^{\mathrm{cd}}$ & $21,1^{\mathrm{b}}$ \\
\hline 6 & 15 & $1,09^{\mathrm{cd}}$ & $0,0165^{\mathrm{bc}}$ & \\
\hline 7 & 17,5 & $1,25^{\mathrm{bc}}$ & $0,0195^{\mathrm{ab}}$ & $26,7^{\mathrm{a}}$ \\
\hline 8 & 20 & $1,42^{\mathrm{b}}$ & $0,0195^{\mathrm{ab}}$ & \\
\hline 9 & 22,5 & $1,76^{\mathrm{a}}$ & $0,0210^{\mathrm{a}}$ & $29,2^{\mathrm{a}}$ \\
\hline
\end{tabular}

a, b... (coluna) - médias seguidas de mesma letra não diferem entre si pelo teste de Tukey $(p>0,05)$. 
TABELA 5 - Coeficientes de correlação linear entre o tempo de fritura e as determinações físico-químicas realizadas no óleo de soja das Fritadeiras I e II.

\begin{tabular}{cccc}
\hline $\mathbf{x}$ & $\mathbf{y}$ & Fritadeira I & Fritadeira II \\
\hline Tempo & Dienos Conjugados & $0,98^{*}$ & $0,98^{*}$ \\
Tempo & TBA & $0,80^{*}$ & $0,80^{*}$ \\
Tempo & Compostos Polares Totais & $0,90^{*}$ & $0,90^{*}$ \\
\hline
\end{tabular}

* significativo $(\mathrm{p}<0,05)$.

\section{CONCLUSÕES}

a) De acordo com os resultados obtidos observouse, de modo geral, que houve um aumento significativo nas alterações do óleo de soja com o tempo de fritura, para ambas as fritadeiras.

b) Diante das condições do processo e dos tipos de alimentos empregados neste estudo, recomenda-se o consumo dos alimentos fritos até 12,5 horas de aquecimento, pois acima deste tempo de fritura, observouse aumento do nível de alteração de alguns compostos, em destaque os compostos polares, que apresentaram valores acima dos recomendados pelas regulamentações de alguns países.

c) Encontrou-se boas correlações entre as determinações físico-químicas e tempo de fritura, sendo 0,98 o melhor coeficiente de correlação obtido entre dienos conjugados e tempo de fritura, para ambas as fritadeiras.

\section{AGRADECIMENTOS}

Ao PIBIC/CNPq pela concessão de bolsa de Iniciação Científica.

\section{REFERÊNCIAS BIBLIOGRÁFICAS}

AMERICAN OIL CHEMISTS SOCIETY.Official methods and recommended practices of the AOCS. 3. ed. Champaign, 1993.

BILLEK, G. Heated fats in the diet. In: PADLEY, F. B.; PODMORE, J. The role of fats in human nutrition. Chichester: E. Horwood, 1985. cap. 12, p. 163-172.

CELLA, R. C. F.; REGITANO-D'ARCE, A. B.; SPOTO, M. H. F. Comportamento do óleo de soja refinado utilizado em fritura por imersão com alimentos de origem vegetal. Ciência e Tecnologia de Alimentos, Campinas, v. 22, n. 2, p. 111-116, 2002.

CUESTA, C.; SÁNCHES-MUNIZ, F. J.; HERNÁNDEZ, I.; VARELA, L. S. Modificaciones de un aceite de oliva durante las frituras sucesivas de patatas: correlaciones entre distintos índices analíticos y de evaluación global de la degradación. Revista de Agroquímica e Tecnologia de Alimentos, [S.1.], v. 31, n. 4, p. 523-531, 1991.

DEL RÉ, P. V. Comportamento de óleos vegetais em frituras descontínuas de produtos pré-fritos congelados. 2003. $121 \mathrm{f}$. Dissertação (Mestrado em Engenharia e Ciência de Alimentos) - Universidade Estadual Paulista, São José do Rio Preto, 2003.

DOBARGANES, M. C.; PÉREZ-CAMINO, M. C.; GONZÁLEZ-QUIJANO, R. G. Métodos analíticos de aplicación en grasas calentadas: I. determinación de ésteres metílicos no alterados. Grasas y Aceites, Sevilla, v. 35, n. 3, p. 172-177, 1984.

DOBARGANES, M. C.; PÉREZ-CAMINO, M. C.; MÁRQUEZ-RUIZ, G. High performance size exclusion chromatography of polar compounds in heated and non heated fats. Fat Science Technology, [S.1.], v. 90, p. 308-311, 1988.

FIRESTONE, D.; STIER, R. F.; BLUMENTHAL, M. M. Regulation of frying fats and oils. Journal of Food Tecnology, Oxford, v. 45, n. 2, p. 90-94, 1991.

GOMES, F. P. Curso de estatística experimental. 14. ed. Piracicaba: Nobel, 2000. 477 p.

INSTITUTO ADOLFO LUTZ. Normas analíticas do Instituto Adolfo Lutz. São Paulo, 1985. p. 245-266.

LAKE, R. J.; SCHOLES, P. Quality and consumption of oxidized lipids from deep-frying fats and oils in New Zealand. Journal of the American Oil Chemistry Society, Chicago, v. 74, n. 9, p. 1065-1068, 1997.

MASSON, L.; ROBERT, P.; IZAURIETA, M.; ROMERO, N.; ORTIZ, J. Fat deterioration in deep fat frying «french fries» potatoes at restaurant and food shop sector. Grasas y Aceites, Sevilla, v. 50, n. 6, p. 460-468, 1999. 
MASSON, L.; ROBERT, P.; ROMERO, N.; IZAURIETA, M.; VALENZUELA, S.; ORTIZ, J.; DOBARGANES, M. C. Comportamiento de aceites poliinsaturados en la preparación de patatas fritas para consumo inmediato: formación de nuevos compuestos y comparación de métodos analíticos. Grasas y Aceites, Sevilla, v. 48, n. 5, p. 273-281, 1997.

PAUL, S.; MITTAL, G. S. Regulating the use of degraded oil/ fat in deep-fat/oil food frying. Critical Reviews in Food Science and Nutrition, Cleveland, v. 37, n. 7, p. 635-662, 1997.

POZO-DÍEZ, R. M. Estudio del proceso de fritura de alimentos frescos y congelados prefritos: comportamiento del aceite de semilla de girasol de alto contenido en acido oleico. 1995. 338 f. Tese (Doutorado em Farmácia)-Facultad de Farmacia, Universidad de Alcalá de Henares, Alcalá de Henares, 1995.
STEVENSON, S. G.; VAISEY-GENSER, M.; ESKIN, N. A. M. Quality control in the use of deep frying oils. Journal of the American Oil Chemistry Society, Chicago, v. 61, n. 6, p. 1102-1108, 1984.

WALTKING, A. E.; WESSELS, H. Chromatographic separation of polar and non-polar components of frying fats. Journal Association Official Chemistry, [S.1.], v. 64, n. 6, p. 1329-1330, 1981.

WHITE, P. J. Métodos para medir los cambios en los aceites de fritura por inmersión en grasas. Alimentaria, [S.1.], n. 9, p. 81-87, 1991.

ZHANG, W. B.; ADDIS, P. B. Prediction of levels of cholesterol oxides in heated tallow by dielectric measurement. Journal of Food Science, Chicago, v. 55, p. 1673-1675, 1990. 